\title{
Controlling inertial focussing using rotational motion
}

\author{
Christopher Prohma ${ }^{\text {, }}$ Nikolas Zöller, and Holger Stark \\ Institute of Theoretical Physics, Technische Universität Berlin, Hardenbergstr. 36, 10623 Berlin, Germany
}

Received 11 February 2014 and Received in final form 3 April 2014

Published online: 15 May 2014

(C) The Author(s) 2014. This article is published with open access at Springerlink.com

\begin{abstract}
In inertial microfluidics lift forces cause a particle to migrate across streamlines to specific positions in the cross section of a microchannel. We control the rotational motion of a particle and demonstrate that this allows to manipulate the lift-force profile and thereby the particle's equilibrium positions. We perform two-dimensional simulation studies using the method of multi-particle collision dynamics. Particles with unconstrained rotational motion occupy stable equilibrium positions in both halfs of the channel while the center is unstable. When an external torque is applied to the particle, two equilibrium positions annihilate by passing a saddle-node bifurcation and only one stable fixpoint remains so that all particles move to one side of the channel. In contrast, non-rotating particles accumulate in the center and are pushed into one half of the channel when the angular velocity is fixed to a non-zero value.
\end{abstract}

\section{Introduction}

The transport of particles in a fluid is a recurring problem on very different length scales. It ranges from dust particle in oil transport to cells in biomedical applications. In all these systems fluid inertia cannot be neglected and inertial migration of particles across streamlines is observed. In particular for biomedical applications a large number of microfluidic devices using inertial migration has been proposed in recent years [1-7]. They are used for cell counting, cell sorting, and mechanical phenotyping.

The devices are carefully designed for controlling the motion of the dispersed colloidal particles. Commonly they use a combination of special shapes for the microchannel cross sections and the action of inertial lift forces to tailor the equilibrium positions of the particles at the channel outlet $[5,6]$. Examples are regular extrusions along the channel to create microscale vortices $[2,3]$, a trapezoidal channel cross section to enhance particle separation [6], or the controlled placement of pillars into the fluid flow to guide particles through the channel [8]. Aside from geometry, microfluidic devices commonly employ external control fields both at low and modest Reynolds numbers Re. Sheath flows are used to focus particles [9] and to analyze the mechanical properties of cells in order to detect cancer [7]. Optical tweezers are employed, in combination with feedback control [10-12] or as optical lattices [13], to enhance particle separation.

This article deals with rotating particles in microfluidic channels. Using the angular momentum of light, op-

\footnotetext{
a e-mail: christopher.prohm@tu-berlin.de
}

tical tweezers operating in the Laguerre-Gaussian mode can transfer angular momentum to birefringent or shape anisotropic particles and rotate them $[14,15]$. Superparamagnetic beads align with an intrinsic axis along an external magnetic field due to a small anisotropy in their magnetic susceptibility [16]. In particular, they can follow a rotating magnetic field and assume its angular velocity [17]. Rotating particles were used to study the mechanical properties of DNA [18] and show rich patterns of self-organization $[19,20]$. Furthermore, rotating colloids were employed to construct micropumps both in experiments [21,22] and simulations [23, 24].

Segré and Silberberg were the first to explicitly attribute cross-streamline migration at intermediate Reynolds number to fluid inertia [25]. The theoretical analysis of inertial effects in microfluidics is complicated by the nonlinear convection term of the Navier-Stokes equations. Analytic studies $[26,27]$ are only partly applicable as they assume particle sizes much smaller than the channel diameter, which is not valid in many microfluidic devices. Here numerical simulation studies are important. Previous investigations used the finite-element method [28,29], lattice-Boltzmann simulations [30,31], and the method of multi-particle collision dynamics [32].

Inertial lift forces are a common means to describe cross-streamline migration of particles in inertial microfluidics. In this article we investigate how the controlled rotational motion of a colloidal particle influences the liftforce profile and thereby the equilibrium positions in the channel cross section. We will perform two-dimensional simulation studies based on the method of multi-particle collision dynamics. When the particle's rotation is not con- 


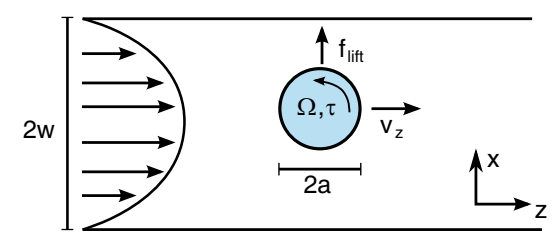

Fig. 1. Two-dimensional channel geometry with width $2 w$. A parabolic Poiseuille flow at Reynolds number Re drives a circular particle with diameter $2 a$. The particle moves with an axial velocity $v_{z}$ and angular velocity $\Omega$. It experiences the inertial lift force $f_{\text {lift }}$ in lateral direction.

strained, it occupies stable equilibrium positions in both halfs of the channel while the center is unstable. We first control particle rotation by an external torque and show that with increasing torque particles only occupy one half of the channel when passing a saddle-node bifurcation. In contrast, non-rotating particles accumulate in the center and are pushed to one side of the channel for non-zero but fixed angular velocity.

The article is organized as follows. In sect. 2 we describe the system geometry and shortly introduce the simulation method. In sect. 3 we review particle motion in the inertial regime without rotation control. We study the effect on the lift force profile when an external torque is applied in sect. 4 and when the angular velocity is controlled in sect. 5. Finally, we close with a summary and concluding remarks in sect. 6 .

\section{Methods}

\subsection{System}

We consider a two-dimensional channel geometry with width $2 w$ and length $L$ as sketched in fig. 1 . The channel is filled by a Newtonian fluid with kinematic viscosity $\nu$ and density $\rho$. We apply a pressure-driven, parabolic Poiseuille flow with maximum flow velocity $u_{\max }$ at the channel center and characterize the relevance of fluid inertia by the channel Reynolds number $\operatorname{Re}=2 u_{\max } w / \nu$. The vorticity $\Omega_{0}(\vec{x})=[\vec{\nabla} \times \vec{u}(\vec{x})]_{y} / 2$ assumes its maximum value at the channel wall and equals $\Omega_{\max }=\nu \operatorname{Re} / 2 w^{2}$. In units of the MPCD method introduced below, the respective channel width and length are $2 w=36$ and length $L=100$. Along the channel axis we implement periodic boundary conditions and choose the coordinate system such that the $z$-axis points along the axial direction and the $x$-axis along the lateral direction.

Inside the channel we place a neutrally buoyant, circular particle with radius $a$. We denote its lateral distance from the center line by $x$. The particle moves with an axial velocity $v_{z}$ along the channel and experiences a lateral lift force $f_{\text {lift }}$ while rotating with an angular velocity $\Omega$.

\subsection{Multi-particle collision dynamics}

To simulate the flow of a Newtonian fluid, we use the method of Multi-Particle Collision Dynamics (MPCD)
[33-35]. MPCD is a mesoscopic Navier-Stokes solver which includes thermal fluctuations. It uses point particles which stream during a time intervall $\Delta t_{c}$ with their respective velocities. Then during the collision step the simulation volume is divided into cells. The velocities of the particles in each cell are redistributed by a collision rule such that linear momentum is preserved. Here we employ a specific variant of MPCD called MPCD-AT $+\mathrm{a}$, which simulates fluid flow at constant temperature and also conserves local angular momentum which is especially important when considering rotating surfaces [36]. Details of our implementation and also how to generate the pressure-driven Poiseuille flow are described in our previous work on inertial microfluidics with three-dimensional flows [32].

In our simulations we choose all quantities in MPCD units. In particular, we use the edge length of the quadratic collision cells $a=1$, the collision time $\Delta t_{c}=0.1$, density $\rho=10$, and temperature $T=1$. These parameters result in a kinematic viscosity $\nu=0.42$ which we calculate from analytic expressions derived in [37]. As already explained in ref. [32], thermal fluctuations are too strong in the simulations. However, by averaging over a sufficient number of realizations, we are able to determine lift-force profiles which we are mainly interested in.

\subsection{Lift-force profiles and probability distributions from MPCD simulations}

To determine lift forces acting on a particle and its axial velocities as a function of particle position, we keep the lateral position $x$ fixed by always setting the lateral velocity $v_{x}$ to zero. The motion along the channel axis and the rotational motion of the particle are not constrained. We determine the lift force $f_{\text {lift }}$ by averaging the momentum transfer from the fluid particles on the colloidal particle in steady state over the last 49000 time steps. Averaging over the same time steps, also gives the steady state axial velocity $v_{z}$ and the angular velocity $\Omega$ of the particle. In sect. 5 we will consider particle motion with constrained angular velocity. Then we also calculate the torque $\tau$ exerted by the fluid onto the particle by averaging the angular momentum transfer in steady state.

We determine the center-of-mass distribution function of the colloidal particle by letting it move without any constraints through the channel. From the particle trajectories we calculate the distribution functions using a kernel density estimate [38] with a bandwidth $b=0.03 w$ as explained in [32].

\section{Uncontrolled rotational motion}

First we illustrate the basic features of particle motion inside a microchannel in the inertial regime at intermediate Reynolds number when particle rotation is not controlled. Due to the inertial lift force, the particle migrates across streamlines counterbalanced by thermal diffusion. As a result, a stationary center-of-mass distribution function $P(x)$ in lateral direction forms, which we plot in fig. 2 . 


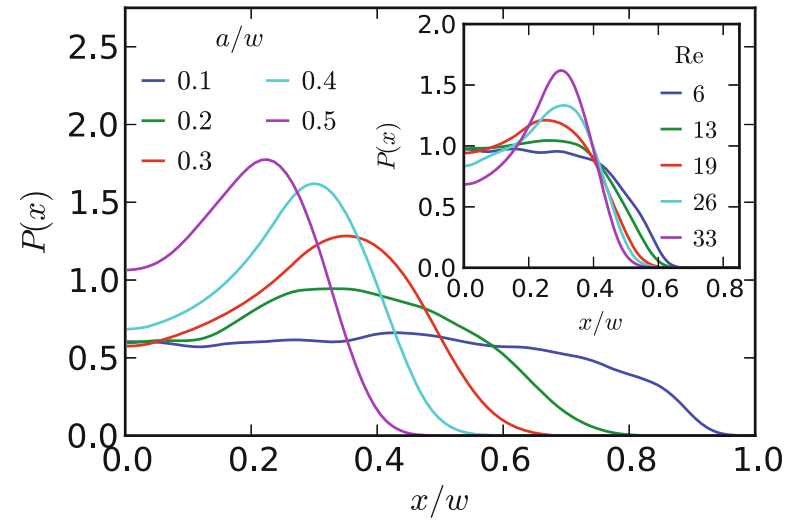

Fig. 2. Lateral center-of-mass distribution function $P(x)$ for different particle sizes $a / w$ at Reynolds number $\operatorname{Re}=33$. Rotational particle motion is not controlled. Inset: lateral distribution function for different Re and $a / w=0.4$.

We observe how $P(x)$ gets narrower and shifts towards the channel center with increasing particle size $a / w$. The peak is located at the position of zero lift force. In addition, the distribution function becomes narrower with increasing Reynolds number as demonstrated in the inset.

In fig. 3 we plot the lift-force profile for particle sizes $a=0.3 w$ and $0.4 w$ at channel Reynolds number $\operatorname{Re}=33$. The channel center at $x=0$ with $f_{\text {lift }}=0$ is an unstable fixpoint since any disturbance drives the particle towards the channel walls. However, close to a wall the particle is pushed towards the channel center. Both effects chancel at about $x \approx \pm 0.3 w$ and a stable equilibrium position with $f_{\text {lift }}=0$ occurs. Furthermore, we observe that the strength of the lift force increases with increasing particle size. The particle rotates with an angular velocity $\Omega$ which is linear in the distance from the center as the inset of fig. 3 shows. Indeed, from Faxen's law [39], we expect $\Omega$ to be equal to the local vorticity in an unconstrained fluid, which for an unperturbed Poiseuille flow is linear in $x$. Small deviations due to hydrodynamic interactions with the bounding walls will be illustrated in more detail in fig. 12. Both, the distribution functions and the lift-force profiles show the same qualitative behavior as in simulations of three-dimensional systems [28,32]. As demonstrated in [32], we can interpret the lateral distribution function as a Boltzmann distribution since the particle performs thermal motion in a potential associated with the inertial lift force.

One can explain the lateral lift force as the result of a difference in dynamic pressure that occurs when in a shear flow the flow velocities close to the particle surface vary [40]. In particular, in this interpretation the inertial lift force is caused by the curvature of the Poiseuille flow profile. The absolute relative velocity between the colloid and fluid is higher on the wall side than on the side facing the channel centerline. This creates a lower dynamic pressure on the wall side and the particle is pushed towards the wall. Following this explanation, we therefore expect that changing the angular velocity of the colloid alters the lift-force profile since the relative velocities on the two colloid sides change in the opposite way. In the

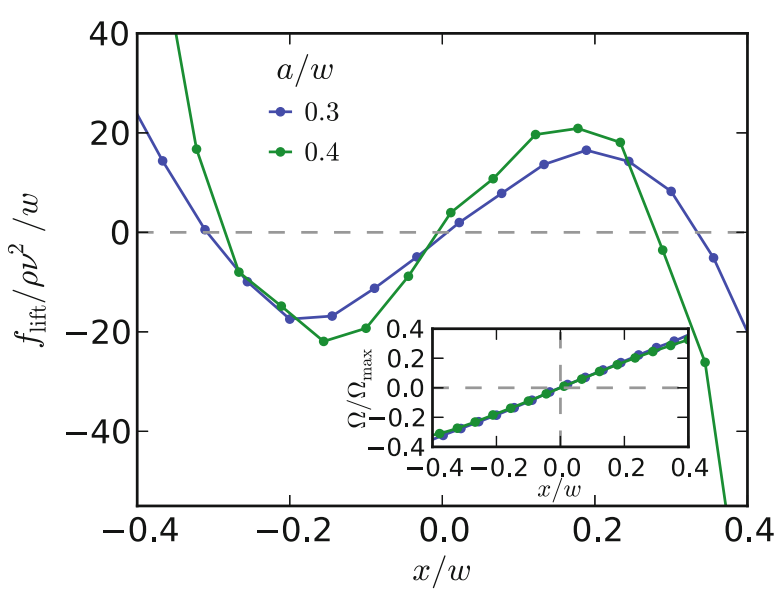

Fig. 3. Lift-force profiles $f_{\text {lift }}$ plotted versus particle position $x$ for two particle sizes $a / w=0.3$ and 0.4 at $\operatorname{Re}=33$. The inset shows the angular velocity $\Omega$ of the particle in units of the maximum vorticity $\Omega_{\max }$ plotted versus $x$.

following two sections we will explore this effect and show how it can be used to control lateral particle migration in the microchannel.

\section{Control by torque}

In the present section we investigate how one can control particle migration by applying a constant torque $\tau$ to the particle. From fig. 4, where we plot the lateral center-ofmass distribution for increasing $\tau$, we observe that the external torque breaks the original mirror symmetry of the microfluidic system. With increasing $\tau$ particles preferentially move to the upper half of the channel $(x>0)$ and ultimately the asymmetric bimodal distribution assumes a unimodal shape. The reason becomes clear with the help of fig. 1. In the lower half of the channel the particle side facing the wall moves with the flow due to the applied torque which decreases the difference in relative flow velocities between both sides of the particle. In the upper half the difference increases and thereby the difference in dynamic pressure becomes larger. This results in a force which pushes the particle against the upper wall.

Modifications in the lift-force profile reflect the change in the distribution function as demonstrated in fig. 5 . With increasing torque the whole force profile is shifted upwards. Stable and unstable equilibrium positions in the lower half of the channel $(x<0)$ move towards each other. They ultimately merge (not shown) and vanish completely which corresponds to the case where the distribution function becomes unimodal. Figure 6 illustrates the corresponding bifurcation scenario by plotting the equilibrium positions $x_{\mathrm{eq}}$ of the fix points versus the applied torque. The stable and unstable fixpoint in the lower half of the channel merge and annihilate each other in a saddle-node bifurcation, while the second stable fixpoint slightly moves towards the wall. We determined the bifuraction scenario directly from the distribution functions in fig. 4 . The un- 


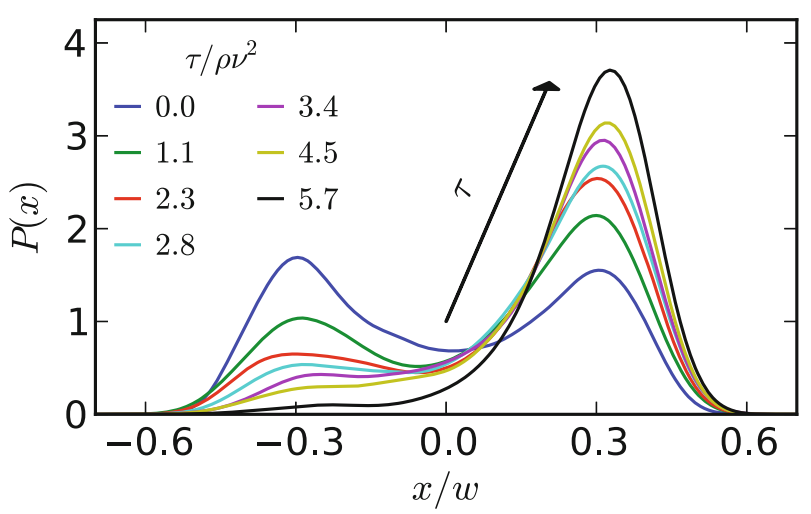

Fig. 4. Distribution with a additional torque imposed on the particle for $\operatorname{Re}=33, a / w=0.4$. The torque breaks the symmetry of the system and leads to a preferential migration in direction of the faster particle side.

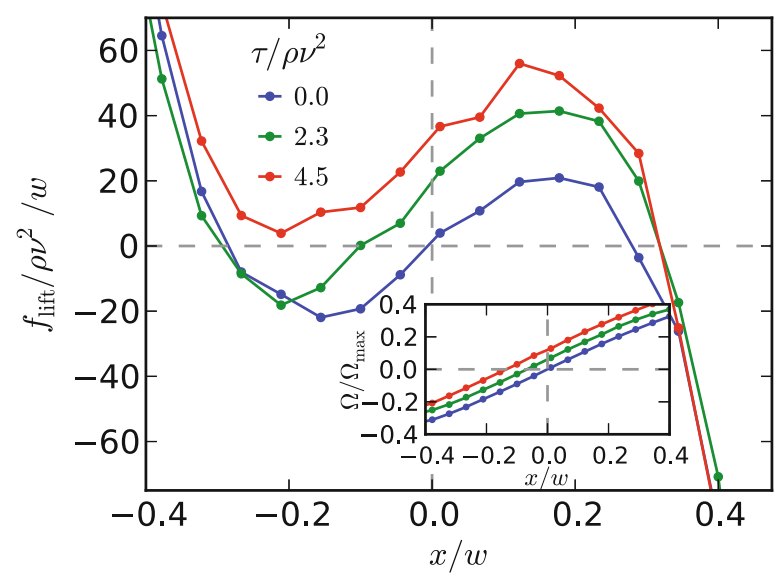

Fig. 5. Lift-force profile for several applied torque values $\tau$ for $a / w=0.4$ and at $\operatorname{Re}=33$. Inset: rotational velocity plotted versus $x$ for the same torque values.

stable fix point corresponds to the minimum in the distribution function while the stable equilibrium positions follow from the maxima.

The inset of fig. 5 shows how the applied torque $\tau$ changes the angular velocity $\Omega$ along the channel cross section. This is well described by Faxén's law [39]

$$
\Omega-\Omega_{0}=\xi_{r}^{-1} \tau .
$$

However, small deviations occur due hydrodynamic interactions with the channel walls, as we will demonstrate in the following section and fig. 12. Here $\Omega_{0}(x)$ is the vorticity of the unperturbed Poiseuille flow and $\xi_{r}=4 \pi \rho \nu a^{2}$ the rotational friction coefficient of a circular disk [41]. In contrast to an equivalent investigation at small $\operatorname{Re} \approx 0[23]$, we do not observe that the axial particle velocity $v_{z}$ varies for different torques and compared to the free particle motion (not shown). We expect this since the external driving by the Poiseuille flow is quite strong.

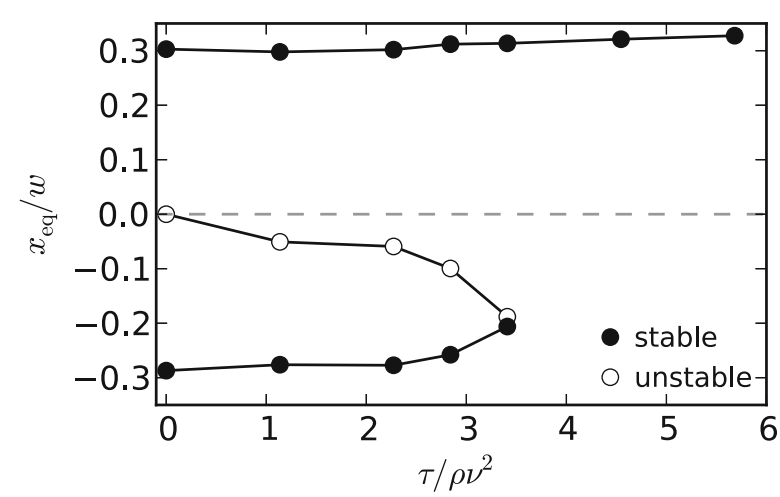

Fig. 6. Bifurcation scenario for the stable and unstable equilibrium positions $x_{\text {eq }}$ plotted as a function of the applied torque $\tau$. The particle size is $a / w=0.4$ and $\operatorname{Re}=33$. A saddle-node bifurcation occurs.

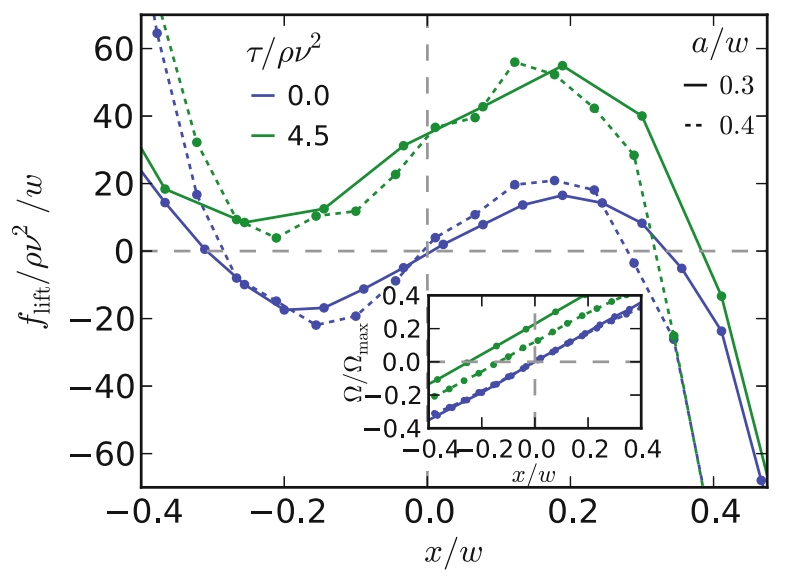

Fig. 7. Lift-force profiles at $\mathrm{Re}=33$ for zero torque (blue) and $\tau=0.12 \xi_{r} \dot{\gamma}_{\max }$ (green) for two particles sizes $a=0.3 w$ (solid) and $a=0.4 \mathrm{w}$ (dashed). Inset: rotational velocity plotted versus $x$ for the same parameters.

In fig. 7 we plot the lift-force profile for zero torque and $\tau=4.5 \rho \nu^{2}$ for two particle sizes $a=0.3 w$ and $0.4 w$. Surprisingly, the applied torque shifts the force profiles for both particle sizes upwards by the same force value. Only close to the wall deviations occur since the torque-induced lift force has to compete with the strong wall-induced lift forces. Since the rotational friction coefficient of the small particle is smaller by a factor $(4 / 3)^{2}=1.77$ compared to the larger particle, we observe a stronger increase in the angular velocity as documented by the inset in fig. 7 . We obtain quantitative agreement with Faxén's law (1) with small deviations when the particle approaches the walls.

The contribution to the lift force generated by the applied torque is an inertial force. In fig. 8, we plot distribution functions for different Reynolds numbers Re all determined with the same applied torque. We observe that with decreasing Re the particle focussing vanishes although the imposed torque stays the same. 


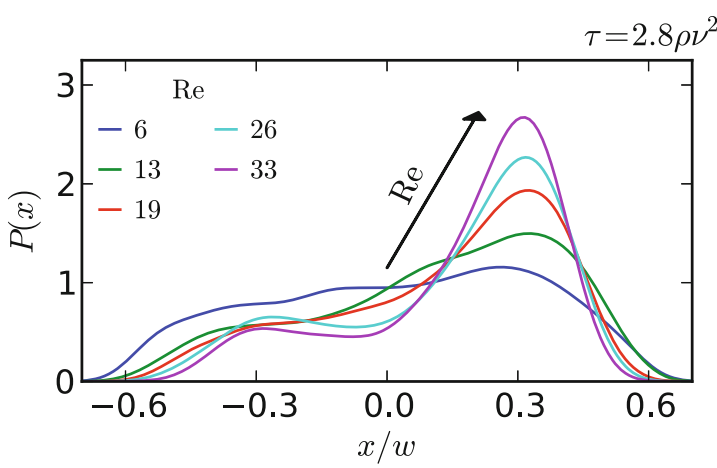

Fig. 8. Distribution functions for a fixed torque value $\tau=$ $2.8 \rho \nu^{2}$ at different Reynolds numbers.

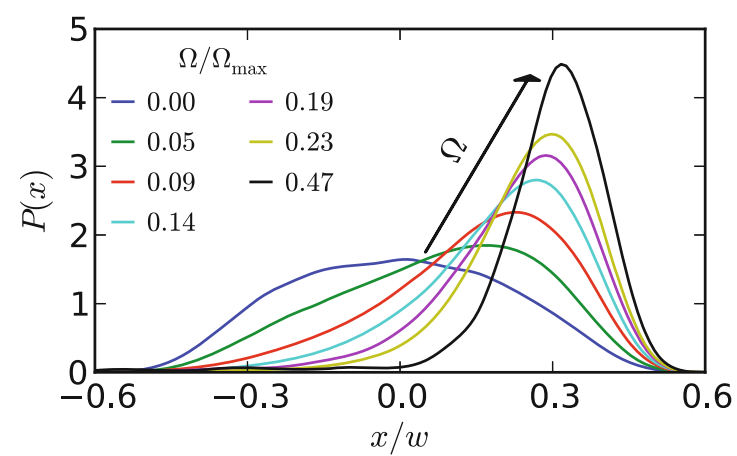

Fig. 9. Particle distribution functions along the lateral direction for different angular velocities $\Omega$ imposed on a particle with radius $a=0.4 w$ at Reynolds number $\operatorname{Re}=33$.

\section{Control by angular velocity}

So far, we controlled the external torque acting on the particles. It adds a constant rotational velocity to the position dependent part $\Omega_{0}$, which is given by the local vorticity of the Poiseuille flow. Now, we control the particles' rotational velocity $\Omega$ and keep it constant in the channel cross section. This will offer new insights. To impose $\Omega$, we think, for example, about superparamagnetic colloids. The magnetic susceptibility is typically anisoptropic and the particles align with their intrinsic axes along an external field [16]. The particle axis can also follow a rotating magnetic field and revolves with the same angular velocity $[17]$.

Figure 9 shows particle distribution functions along the lateral direction for different angular velocities $\Omega$. The particle has a radius $a=0.4 \mathrm{w}$ and the Reynolds number is $\mathrm{Re}=33$. We observe that the distribution $P(x)$ is unimodal for all angular velocities in contrast to the bimodal distribution when the particle rotation is not controlled. At $\Omega=0$ the particle predominantly stays in the center of the channel. With increasing angular velocity the maximum of $P(x)$ shifts towards the wall, in agreement with our findings for the lift-force profile discussed below and illustrated in fig. 11. These results show that particle rotation is important for inertial focussing. In contrast to findings in [28], we are even able to focus the parti-

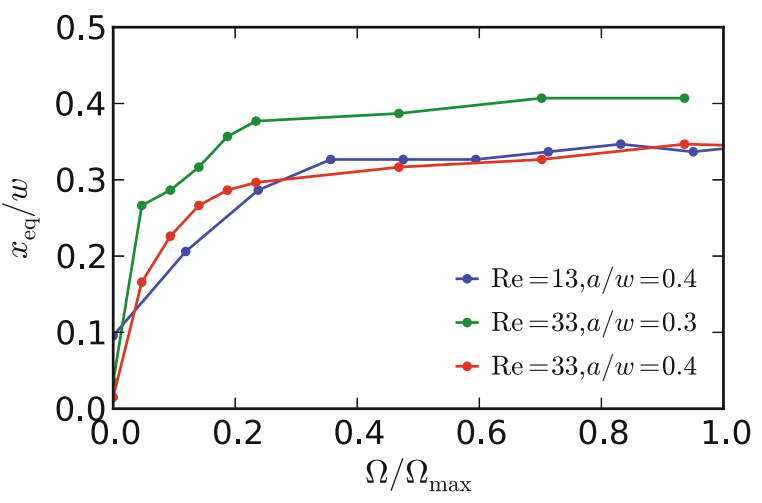

Fig. 10. Equilibrium position $x_{\mathrm{eq}}$ as a function of the imposed angular velocity $\Omega$ for different particle sizes and Re.

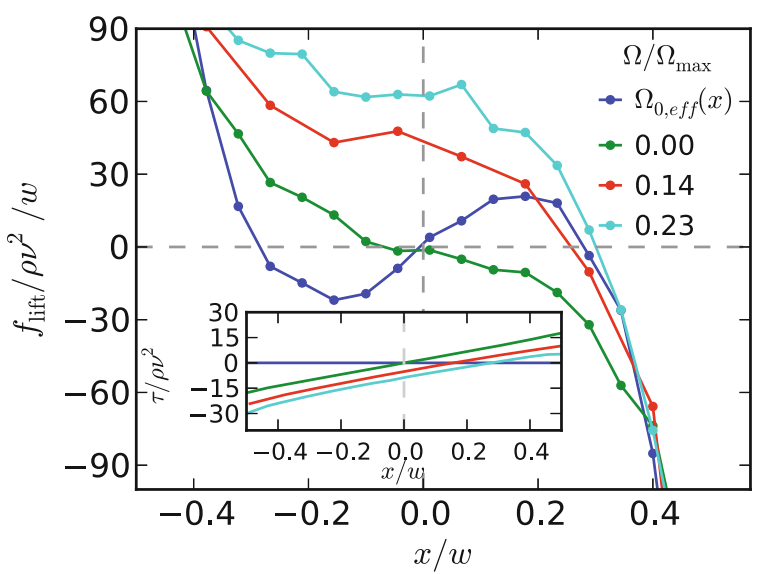

Fig. 11. Lift-force profiles for several imposed angular velocities $\Omega$ and the unconstrained motion with $\Omega=\Omega_{0, \text { eff }}(x)$. Inset: torque necessary to impose a given angular velocity at different lateral positions $x$.

cle to the channel center. Finally, we note that a negative $\Omega$ shifts the particle into the lower half of the channel. So within inertial microfluidics on can position particles inside the channel by controlling their angular velocity.

To be more quantitative, we determine the equilibrium position $x_{\text {eq }}$ from the maximum of the distribution function and in fig. 10 plot it versus the angular velocity $\Omega$ for different particle sizes and Reynolds numbers. Starting from the center for zero angular velocity, the particle position shifts closer to the wall with increasing $\Omega$, as already stated. Similar to the unconstrained case, the equilibrium position of the smaller particle is always closer to the wall as the green $(a=0.3 w)$ and the red $(a=0.4 w)$ graph illustrate. We also plot the equilibrium position of a particle with radius $a / w=0.4$ at a lower Reynolds number $\operatorname{Re}=13$. Since we rescale $\Omega$ by the maximum vorticity $\Omega_{\max } \propto \mathrm{Re}$, the absolute values of the imposed angular velocities are only $40 \%$ of the ones at the larger Reynolds number. Nevertheless, with this scaling we observe a similar behavior of the equilibrium positions for both Reynolds numbers. 


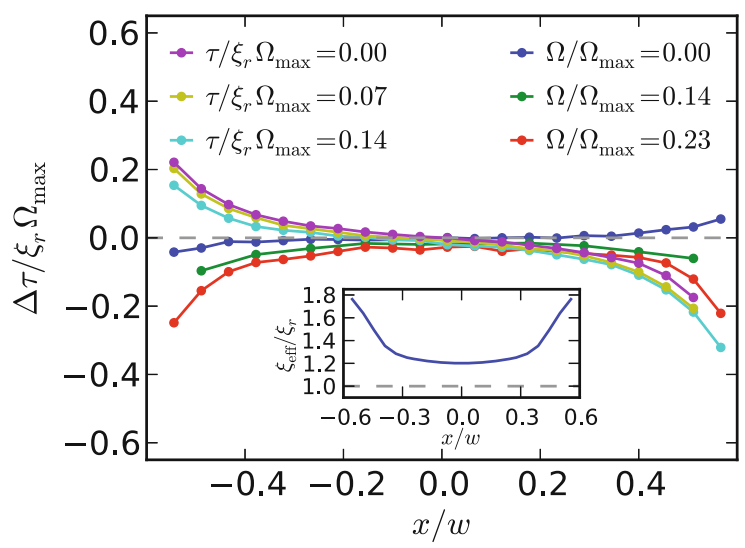

Fig. 12. The deviation $\Delta \tau=\tau-\xi_{r}\left(\Omega-\Omega_{0}\right)$ from the torque expected according to Faxén's law, plotted either for prescribed torque $\tau$ or angular velocity $\Omega$ for $a / w=0.4$, $\operatorname{Re}=33$. Inset: the effective rotational friction coefficient $\xi_{\text {eff }}=\tau /\left(\Omega-\Omega_{0, \text { eff }}\right)$ plotted versus particle position.

Figure 11 shows how the inertial lift-force profile is influenced by the imposed constant angular velocity $\Omega$. For comparison, we also show the lift-force profile of an unconstrained particle which has an angular velocity of $\Omega_{0, \mathrm{eff}}(x)$ almost equal to the vorticity of the Poiseuille flow $\Omega_{0}$, as mentioned earlier. When we prevent the particle from rotating, it experiences a force which always drives it back to the channel center. With increasing angular velocity, the equilibrium position shifts towards the wall in agreement with our findings for the distribution function. Close to the wall the wall lift force dominates and all curves fall on top of each other. We observe similar behavior for other particle sizes and Reynolds numbers (not shown). The inset shows the external torque necessary to impose the constant $\Omega$. Clearly, for $\Omega=0$, the torque has to compensate the rotational motion induced by the vorticity $\Omega_{0}$ of the Poiseuille flow $\tau=\xi_{r} \Omega_{0}$. The straight line is then shifted upwards by $\Delta \tau=\xi_{r} \Omega$.

In the end we test the validity of Faxén's law as formulated in eq. (1) with the vorticity of the unperturbed Poiseuille flow. Through hydrodynamic interactions with the channel walls additional flow fields are initiated, which contribute to the flow vorticity. We thereby expect deviations from Faxén's law, when the particle comes close to the wall. To illustrate this, we plot in fig. 12 the deviation from Faxén's law, $\Delta \tau=\tau-\xi_{r}\left(\Omega-\Omega_{0}\right)$, along the $x$ direction. We either fix particle torque $\tau$ and determine particle rotation $\Omega$ by Faxén's law in eq. (1) or vice versa. Clear deviations, when the particle approaches the walls, are visible. The deviations are due to two effects. The real rotational velocity $\Omega_{0 \text {,eff }}$ of the unconstrained particle deviates from $\Omega_{0}$ and the particle experiences an increased rotational friction, in particular, close to the channel walls. We determine an effective friction coefficient by averaging $\xi_{\text {eff }}=\tau /\left(\Omega-\Omega_{0, \text { eff }}\right)$ over all data sets and plot it in the inset of fig. 12. We observe a pronounced increase as the particle approaches the wall.

\section{Conclusion}

In this article we investigated the motion of a colloidal particle in a two-dimensional channel geometry subject to a pressure-driven Poiseuille flow at intermediate Reynolds numbers. We performed a simulation study using multiparticle collision dynamics. We focused on how control of the particle's rotation modifies the lift-force profile and thereby the particle's equilibrium positions in the channel cross section. For uncontrolled particle motion we observed a strong influence of particle size on the equilibrium position in accordance with previous results in three dimensions $[28,42]$. We also discussed the origin of the lift force. In a parabolic flow the fluid velocities in the frame of the colloid differ for the two particle sides facing either the wall or the channel center which creates a dynamic pressure difference [40]. Since the rotational velocity of the particle directly influences the fluid flow, we argued that a control of particle rotation also modies the lift-force profile.

To test this prediction, we first applied a constant torque to the particle. This breaks the mirror symmetry with respect to the channel axis and the particle preferentially migrates to one channel half. With increasing torque, the lift-force profile is shifted upwards to larger values meaning that the positions of the unstable and stable fixpoint in the lower channel half move towards each other until they annihilate in a saddle-node bifurcation and only the stable fixpoint in the upper channel half remains. Second, we directly controlled the particle's angular velocity which in experiments can be achieved with a rotating magnetic field. When the particle does not rotate, it stays in the channel center, and with non-zero rotational velocity it moves into one half of the channel. This offers an interesting possibility to effectively separate non-magnetic and magnetic particles from each other by controlling the rotational velocity of the latter particle type. The response of the particle to an applied torque or a controlled rotational velocity agrees well with Faxén's law. Deviations occur due to the bounding walls which modify the unconstrained rotation of the particle and its rotational friction coefficient.

After having explored the main effect of controlled particle rotation, we will extend our investigations to threedimensional channel geometries. Here, more stable and unstable fixpoints occur [31] and it will be interesting to check how they are modified under controlled rotation. Furthermore, at low Reynolds number dense suspensions of colloids subject to a constant torque show very interesting collective behavior [23]. When we combine this with the spontaneous ordering of particles into one-dimensional crystals at moderate Reynolds number due to the presence of inertial lift forces $[43,44]$, we expect a very rich and interesting collective behavior, which we plan to explore.

We acknowledge financial support by the Deutsche Forschungsgemeinschaft in the framework of the collaborative research center SFB 910. 
Open Access This is an open access article distributed under the terms of the Creative Commons Attribution License (http://creativecommons.org/licenses/by/4.0), which permits unrestricted use, distribution, and reproduction in any medium, provided the original work is properly cited.

\section{References}

1. D. Di Carlo, Lab Chip 9, 3038 (2009).

2. J.-S. Park, S.-H. Song, H.-I. Jung, Lab Chip 9, 939 (2009).

3. A.J. Mach, J.H. Kim, A. Arshi, S.C. Hur, D. Di Carlo, Lab Chip 11, 2827 (2011).

4. S.C. Hur, N.K. Henderson-MacLennan, E.R. McCabe, D. Di Carlo, Lab Chip 11, 912 (2011).

5. A.J. Mach, D. Di Carlo, Biotechnol. Bioeng. 107, 302 (2010).

6. G. Guan, L. Wu, A.A. Bhagat, Z. Li, P.C. Chen, S. Chao, C.J. Ong, J. Han, Sci. Rep. 3, 1475 (2013).

7. J.S. Dudani, D.R. Gossett, T. Henry, D. Di Carlo, Lab Chip 13, 3728 (2013).

8. H. Amini, E. Sollier, M. Masaeli, Y. Xie, B. Ganapathysubramanian, H.A. Stone, D. Di Carlo, Nat. Commun. 4, 1826 (2013).

9. X. Xuan, J. Zhu, C. Church, Microfluid. Nanofluid. 9, 1 (2010).

10. R.W. Applegate Jr., D.N. Schafer, W. Amir, J. Squier, T. Vestad, J. Oakey, D.W. Marr, J. Opt. A - Pure Appl. Op. 9, S122 (2007).

11. X. Wang, S. Chen, M. Kong, Z. Wang, K.D. Costa, R.A. Li, D. Sun, Lab Chip 11, 3656 (2011).

12. M.S. Munson, J.M. Spotts, A. Niemistö, J. Selinummi, J.G. Kralj, M.L. Salit, A. Ozinsky, Lab Chip 10, 2402 (2010).

13. M. MacDonald, G. Spalding, K. Dholakia, Nature 426, $421(2003)$

14. D.G. Grier, Nature 424, 810 (2003).

15. M. Padgett, R. Di Leonardo, Lab Chip 11, 1196 (2011).

16. K. Neuman, T. Lionnet, J.-F. Allemand, Annu. Rev. Mater. Res. 37, 33 (2007).

17. X. Janssen, A. Schellekens, K. Van Ommering, L. Van Ijzendoorn, M. Prins, Biosensors Bioelectron. 24, 1937 (2009).
18. J. Lipfert, J.W. Kerssemakers, T. Jager, N.H. Dekker, Nat. Methods 7, 977 (2010).

19. B.A. Grzybowski, H.A. Stone, G.M. Whitesides, Nature 405, 1033 (2000).

20. B.A. Grzybowski, G.M. Whitesides, Science 296, 718 (2002).

21. S. Bleil, D.W. Marr, C. Bechinger, Appl. Phys. Lett. 88, 263515 (2006).

22. K. Ladavac, D. Grier, Opt. Express 12, 1144 (2004).

23. I.O. Götze, G. Gompper, EPL 92, 64003 (2010).

24. I.O. Götze, G. Gompper, Phys. Rev. E 84, 031404 (2011).

25. G. Segré, A. Silberberg, Nature 189, 209 (1961).

26. B.P. Ho, L.G. Leal, J. Fluid Mech. 65, 365 (1974).

27. E.S. Asmolov, J. Fluid Mech. 381, 63 (1999).

28. D. Di Carlo, J.F. Edd, K.J. Humphry, H.A. Stone, M. Toner, Phys. Rev. Lett. 102, 094503 (2009).

29. J. Feng, H.H. Hu, D.D. Joseph, J. Fluid Mech. 277, 271 (1994).

30. B. Chun, A.J.C. Ladd, Phys. Fluids 18, 031704 (2006).

31. C. Prohm, H. Stark, to be published in Lab Chip (2014), DOI: 10.1039/C4LC00145A.

32. C. Prohm, M. Gierlak, H. Stark, Eur. Phys. J. E 35, 1 (2012).

33. A. Malevanets, R. Kapral, J. Chem. Phys. 110, 8605 (1999).

34. G. Gompper, T. Ihle, D. Kroll, R. Winkler, in Advanced Computer Simulation Approaches for Soft Matter Sciences III, Advances in Polymer Science (Springer, Berlin, Heidelberg, 2008) pp. 1-87.

35. R. Kapral, Adv. Chem. Phys. 140, 89 (2008).

36. I.O. Götze, H. Noguchi, G. Gompper, Phys. Rev. E 76, 046705 (2007).

37. H. Noguchi, G. Gompper, Phys. Rev. E 78, 016706 (2008).

38. B.W. Silverman, Density Estimation (Chapman and Hall, 1986).

39. H. Brenner, J. Fluid Mech. 111, 197 (1981).

40. J.P. Matas, J.F. Morris, E. Guazzelli, Oil Gas Sci. Technol. 59, 59 (2004).

41. A.T. Chwang, T. Wu, J. Fluid Mech 63, 607 (1974).

42. C. Prohm, F. Tröltzsch, H. Stark, Eur. Phys. J. E 36, 1 (2013).

43. W. Lee, H. Amini, H.A. Stone, D. Di Carlo, Proc. Natl Acad. Sci. U.S.A. 107, 22413 (2010).

44. K.J. Humphry, P.M. Kulkarni, D.A. Weitz, J.F. Morris, H.A. Stone, Phys. Fluid 22, 081703 (2010). 